\title{
A Study on the Sound Insulation Effect of Resilient Interlayer of a Floating Floor
}

\author{
Xianfeng Huang ${ }^{1,2, a}$, Chenhui Zhu' 2, b ${ }^{1,}$ Meng Yao, 2, c , and Shi Quan ${ }^{1,2, d}$ \\ ${ }^{1}$ Guangxi Key Laboratory of Disaster Prevention and Engineering Safety, Guangxi University \\ Nanning, 530004, China \\ ${ }^{2}$ College of Civil Engineering and Architecture, Guangxi University, Nanning, 530004, China \\ a email: seulee@163.coml, bemail: huihui8905@qq.com, ${ }^{\mathrm{c}}$ email: 1148500367@qq.com
}

Keywords: Floating floor; Resilient interlayer; Impact sound; Sound insulation

\begin{abstract}
Due to favorable sound isolation to the impact sound transmission, People are prone to adopt floating floor as insulation structure. Of the researches on impact sound insulation through a floating floor, the theoretical study is much less than the experiment analysis. For the purpose to determine sound insulation and the dominate sound transmission path through a floating floor, the Statistical Energy Analysis(SEA) is employed to set up a theoretical model for predicting sound transmission across two floor plates connected by a resilient interlayer, and there are two main paths: the stud path and the cavity path. It reveals that the resilient interlayer plays a key role in impact sound insulation of a floating floor, and there exists a good agreement between predicted and measured sound pressure level (SPL) in the rooms below the floors.
\end{abstract}

\section{Introduction}

Floating floor are situated in multilayer floor and employed to reduce the impact sound, such kind of structure-borne noise resulting from walking or moving the furniture on the floor, and decoration progress[1]. When impacted, the floor vibrations are transmitted through the contact members and impact sound transmits into the next layer rooms creates noise disturbing to the occupant there. Floating floor is the structure that a wood floor on studs separated from a structural concrete floor by a resilient layer (see Fig1), which exhibits a significant effect on isolating the impact sound. It is found that the sound transfer coefficient through a junction will be evidently attenuated by adding the resilient layer[2]. The bending wave model of Craik [3] was developed to predict the effect of the resilient layers. The impact sound through a floating floor at low frequencies was developed by Tongjun Cho[4]. His further research[5] was associated with the prediction on floating floor sound isolation performance via numerical simulation of FEA model and SEA method at low frequencies, and validated the results by experimental method. K. Kim[6] also studied the sound insulation effect of a floating floor below the frequency of $63 \mathrm{~Hz}$, and found that the low stiffness coefficient of elastic cushion, is beneficial to sound insulation of the floor. Hua Tan [7] analyzed the floating floor with resilient cushion and indicated the solid wood floor and floating wood floor by using qualitative theoretical analysis founding that the resilient layer have evident effect on the attenuating the impact sound up to $23 \mathrm{~dB}$, comparing to other floor without resilient interlayer. In this paper, the influence of resilient interlayer on the sound insulation is investigated. As a consequence, theoretical approaches and SPL in the rooms below the floating floor is achieved.

\section{Model Description}

A model to predict structure coupling between parallel panels connected by a resilient layer as shown in Fig.1. The common tie between the timber floor and the structural concrete floor regarded in here is the stud and resilient layer (such as rubbers, polymer foams). And the SEA model involves six subsystems as shown in Fig. 2 


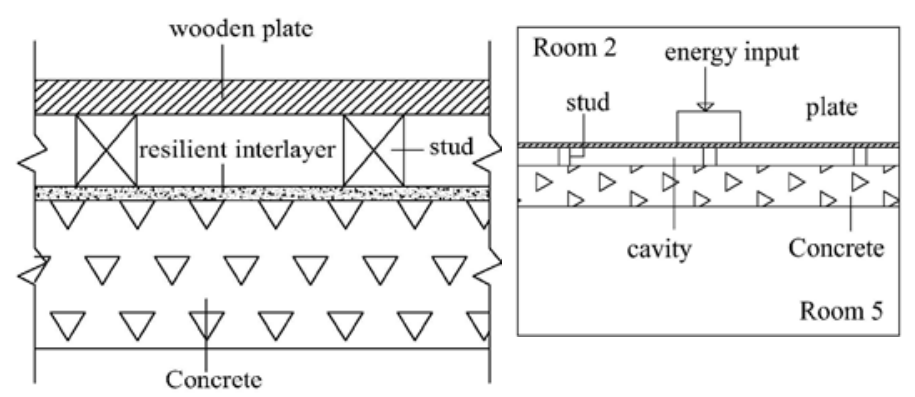

Fig.1. The model of floating floor

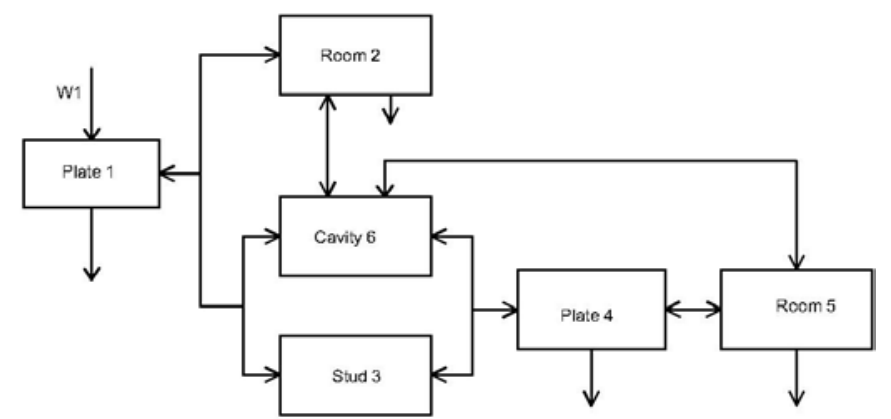

Fig.2. The SEA model of floating floor

The following two paths are the sound transmission road in the system:

Stud path: plate 1-stud 3-interlayer-Concrete 4-room 5;

Cavity path: plate 1-cavity6 - Concrete 4-room 5.

The sound power balance equation [11] for two paths can be as:

Stud path:

$\left[\begin{array}{cccc}-\eta_{1} & 0 & 0 & 0 \\ \eta_{13} & -\eta_{3} & 0 & 0 \\ 0 & \eta_{34} & -\eta_{4} & 0 \\ 0 & 0 & \eta_{45} & -\eta_{5}\end{array}\right]\left[\begin{array}{l}E_{1} \\ E_{3} \\ E_{4} \\ E_{5}\end{array}\right]=\left[\begin{array}{c}-W_{1} / \omega \\ 0 \\ 0 \\ 0\end{array}\right]$

Cavity path:

Resonant:

$\left[\begin{array}{cccc}-\eta_{1} & 0 & 0 & 0 \\ \eta_{16} & -\eta_{6} & 0 & 0 \\ 0 & \eta_{64} & -\eta_{4} & 0 \\ 0 & 0 & \eta_{45} & -\eta_{5}\end{array}\right]\left[\begin{array}{c}E_{1} \\ E_{6} \\ E_{4} \\ E_{5}\end{array}\right]=\left[\begin{array}{c}-W_{1} / \omega \\ 0 \\ 0 \\ 0\end{array}\right]$

Non-resonant:

$\left[\begin{array}{ccc}-\eta_{1} & 0 & 0 \\ \eta_{16} & -\eta_{6} & 0 \\ 0 & \eta_{65} & -\eta_{5}\end{array}\right]\left[\begin{array}{l}E_{1} \\ E_{6} \\ E_{5}\end{array}\right]=\left[\begin{array}{c}-W_{1} / \omega \\ 0 \\ 0\end{array}\right]$

So, the acceleration difference between floor (subsystem 1) to RC slabs (subsystem 4) is:

$D_{a 14, \text { stud }}=10 \log \frac{\eta_{3} \eta_{4} m_{4}}{\eta_{13} \eta_{34} m_{1}}$

The impact sound level through the stud path gives:

$L_{p, \text { stud }}=10 \log \left(\frac{p^{2}}{p_{0}^{2}}\right)=L_{w, 1}+10 \log \left(\frac{\eta_{13} \eta_{34} \eta_{45}}{\eta_{1} \eta_{3} \eta_{4}}\right)-10 \log \left(\frac{V}{T}\right)+14$

The acceleration difference through the cavity path is: 
$D_{a 14, \text { air }}=10 \log \frac{\eta_{6} \eta_{4} m_{4}}{\eta_{16} \eta_{64} m_{1}}$

The impact sound level through the cavity path is:

$\left\{\begin{array}{cc}L_{\mathrm{p}, \text { air }}=10 \times \log _{10}\left(10^{\frac{L_{\mathrm{p}, \text { air non }}}{10}}+10^{\frac{L_{\mathrm{p}, \text { air res }}}{10}}\right) & f \leq f_{c} \\ L_{\mathrm{p}, \text { air }}=L_{\mathrm{p}, \text { air, res }} & f>f_{c}\end{array}\right.$

The critical coincidence frequency,

$$
f_{c, \text { conc }}=\frac{c_{0}^{2}}{2 \pi} \sqrt{\frac{12 \rho_{s}\left(1-\mu^{2}\right)}{E h^{3}}}=\frac{c_{0}^{2} \sqrt{3}}{\pi h c_{L}}
$$

The non-resonant impact sound level through the cavity path is:

$$
L_{\mathrm{p}, \text { air non }}=10 \log \left(\frac{p^{2}}{p_{0}^{2}}\right)=L_{w, 1}+10 \log \left(\frac{\eta_{16} \eta_{65}}{\eta_{1} \eta_{6}}\right)-10 \log \left(\frac{V}{T}\right)+14
$$

And the resonant impact sound level through the cavity path gives:

$$
L_{\mathrm{p}, \text { air, res }}=10 \log \left(\frac{p^{2}}{p_{0}^{2}}\right)=L_{w, 1}+10 \log \left(\frac{\eta_{16} \eta_{64} \eta_{45}}{\eta_{1} \eta_{6} \eta_{4}}\right)-10 \log \left(\frac{V}{T}\right)+14
$$

So, The impact sound level underneath a floating floor is:

$$
L_{p, \text { impact }}=10 \log \frac{1}{10^{-\frac{L_{p, \text { air }}}{10}}+10^{-\frac{L_{p, \text { stud }}}{10}}}
$$

\section{Mechanism of Resilient Interlayer}

Fig. 3 shows the floating floor structure regarded in this paper.

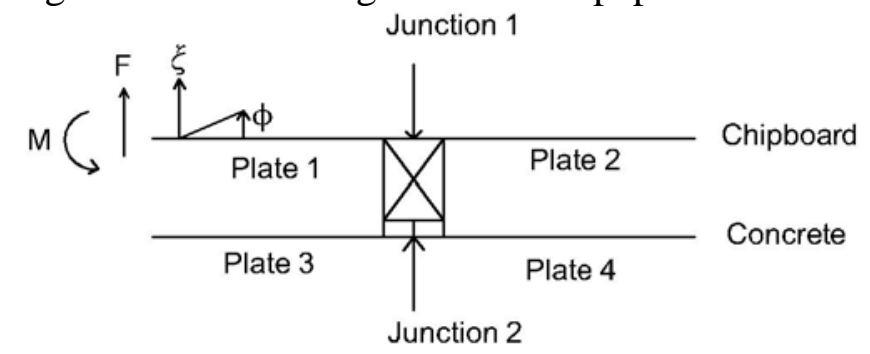

Fig. 3. A theoretical model of floating floor

Each floor plate generates two kinds of waves -- propagating wave and near field wave, define propagating wave amplitude $T_{i}$ and field wave $T_{n i}$, the displacement of plate can be expressed as [11]:

$$
\begin{aligned}
& \xi_{1}=\left(\mathrm{e}^{-i k_{1} \cos \theta_{1} x}+T_{1} \mathrm{e}^{i k_{1} \cos \theta_{1} x}+T_{n 1} \mathrm{e}^{k_{n 1} x}\right) \mathrm{e}^{-i k_{1} \cos \theta_{1} y} e^{i \omega t} \\
& \xi_{2}=\left(T_{2} \mathrm{e}^{-i k_{2} \cos \theta_{2} x}+T_{n 2} \mathrm{e}^{-k_{n 2} x}\right) \mathrm{e}^{-i k_{2} \cos \theta_{2} y} e^{i \omega t} \\
& \xi_{3}=\left(T_{3} \mathrm{e}^{i k_{3} \cos \theta_{3} x}+T_{n 3} \mathrm{e}^{k_{n 3} x}\right) \mathrm{e}^{-i k_{3} \cos \theta_{3} y} e^{i \omega t} \\
& \xi_{4}=\left(T_{4} \mathrm{e}^{-i k_{4} \cos \theta_{4} x}+T_{n 4} \mathrm{e}^{-k_{n}{ }^{x}}\right) \mathrm{e}^{-i k_{4} \cos \theta_{4} y} e^{i \omega t}
\end{aligned}
$$

Where $k_{n i}$ is the near field wave number, and the relationship of angular displacement, bending moment and internal force between each floor can be explained by

$$
\begin{aligned}
& \phi=\partial \xi / \partial x \\
& M=-B\left(\frac{\partial^{2} \xi}{\partial x^{2}}+\mu \frac{\partial^{2} \xi}{\partial y^{2}}\right) \\
& F=B\left[\frac{\partial^{3} \xi}{\partial x^{3}}+(2-\mu) \frac{\partial^{3} \xi}{\partial x \partial y^{2}}\right]
\end{aligned}
$$


There exist resisting moments result from the rotary inertial $M_{c}$ and torsional stiffness of a stud $\frac{\partial M_{y}}{\partial y}$ and force $F_{c}, \frac{\partial F_{y}}{\partial y}$ will be generated in a junction, giving:

$$
\begin{aligned}
& M_{c}+\frac{\partial M_{y}}{\partial y}=\left(-\omega^{2} \mathrm{~J}+T_{T} k_{2}^{2} \sin ^{2} \theta_{2}\right) \phi_{2} \\
& F_{c}+\frac{\partial F_{y}}{\partial y}=\left(-\omega^{2} m^{\prime}+B_{c} k_{2}^{4} \sin ^{4} \theta_{2}\right) \xi_{2}
\end{aligned}
$$

Where $J$ and $T_{T}$ are the polar moment of inertia per unit length and the torsional stiffness of the stud per unit length respectively. In junctions 1 and 2, there are displacement, angular displacement, bending moment and the internal force equilibrium under the boundary conditions. Damping in the interlayer can be expressed the Young's modulus complex times $\left(1+i \eta_{k}\right)$ is $\left(1+i \eta_{k}\right) E^{\prime}, \eta_{k}$ is the loss factor of the resilient interlayer. these equilibrium equations are solved numerically to determined the amplitudes of bending wave on each plate. Then The angle average transmission coefficients from plate 1 to any other plate $j$ is [8]:

$$
\tau_{1 i}=\int_{0}^{\frac{\pi}{2}} \frac{\rho_{s i} k_{1} \cos \theta_{i}}{\rho_{s 1} k_{i} \cos \theta_{1}} \cos \theta \mathrm{d} \theta
$$

Internal force and moment coupling of a boundary are neglected for their contribution less than $0.1 \mathrm{~dB}$ [8]. Sound transmission coefficient from plate1 to the RC slabs is:

$$
\begin{gathered}
\tau_{\text {boardconc }}=\frac{\chi \psi k_{\text {conc }} K^{2}}{2 k_{\text {board }}\left(\chi^{2} \psi^{2} K^{2}+2 \chi \psi K^{2}+K^{2}-4 \chi^{2} \psi^{2} K B_{\text {conc }} k_{\text {conc }}^{3}\right.} \\
\left.-4 K B_{\text {conc }} \chi \psi k_{\text {conc }}^{3}+8 B_{\text {conc }}^{2} \chi^{2} \psi^{2} k_{\text {conc }}^{6}\right)
\end{gathered}
$$

The elastic stiffness per unit length of the resilient layer by Gosele model [9][10].

$$
K=\omega \rho c_{1} b
$$

Where $b$ represents the width of stud, $\rho$ is the density of elastic interlayer, $c_{1}$ represents the longitudinal of the resilient layer.

\section{Insulation effect of interlayer}

The resilient interlayer has a significant effect on attenuating sound transmission through the stud path. The insulation effect of a interlayer depends on elastic stiffness, thickness and density of resilient interlayer. The thickness of resilient interlayer is $5 \mathrm{~mm}$, the density is $40 \mathrm{~kg} / \mathrm{m}^{3}$, and the size of stud is $0.045 \times 0.045 \mathrm{~m}$. The value of stiffness $\mathrm{K}$ are $10^{5}, 10^{6}, 10^{7}, 10^{8} \mathrm{~N} / \mathrm{m}^{2}$ and infinite, separately.

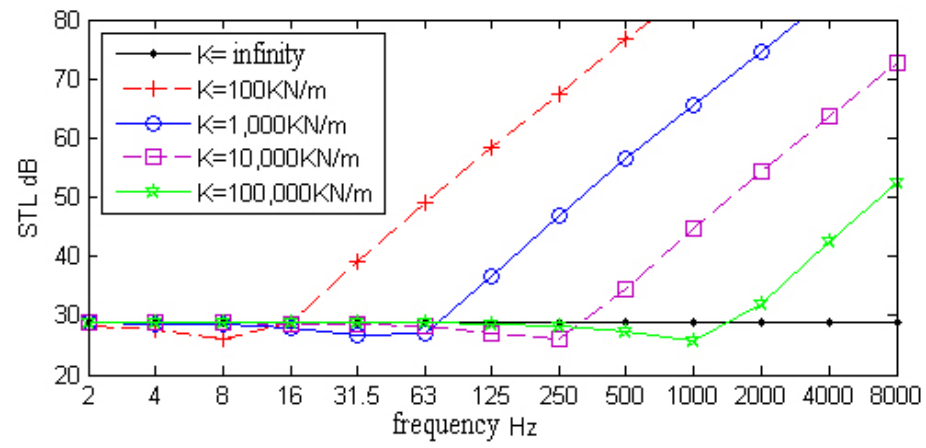

Fig. 4. Sound transmission loss with different stiffness of interlayer

The lower elastic stiffness result in higher sound transmission loss through the stud path as shown in Fig. 4, then, the sound bridge effect is abated due to the insulation of the interlayer. At higher frequencies, sound transmission loss increase rapidly, as the longitudinal wave can not ignore. While three kinds of interlayer with different thickness within 5-30 mm are chosen, the sound transmission through the stud path displays in Fig.5. 


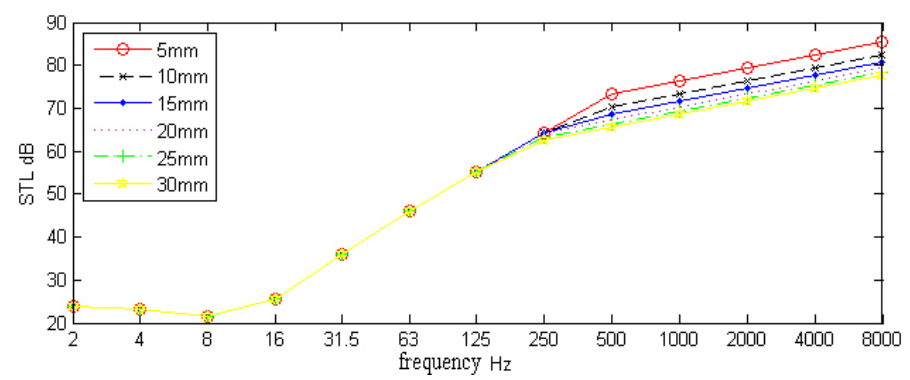

Fig. 5. Sound transmission loss with different thickness of a foam-laminated fabric interlayer

While the frequency below the transition frequency, the interlayer thickness has barely effect on sound transmission loss. Whereas, as the frequency exceeds the transition frequency, The sound transmission loss increases slightly with the thickness.

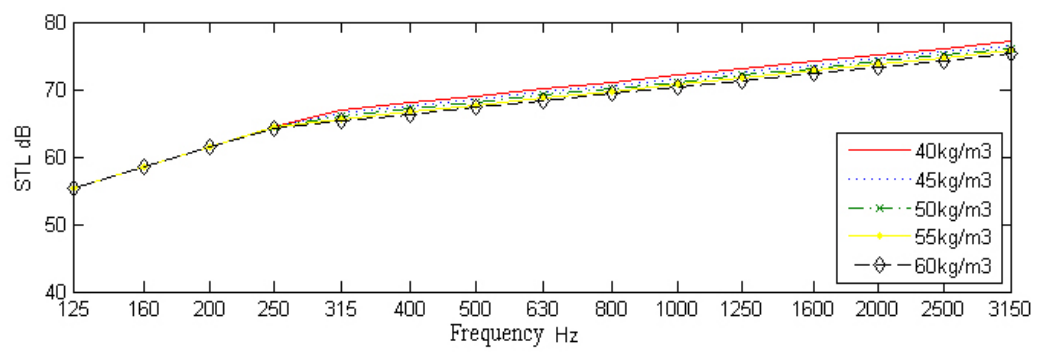

Fig. 6 Relationship between sound transmission loss and interlayer density

As shown in Fig. 6, it can be seen that the sound transmission loss decrease with the density of interlayer. To some extend, low-density interlayer might be beneficial to sound insulation of a floating floor structure, but, this improvement is marginal.

\section{Results}

The floor with a $20 \mathrm{~mm}$ closed-cell foam material $\mathrm{K}=2800$ interlayer and the floor without interlayer are calculated, and the floor slabs are $200 \mathrm{~mm}$ thickness concrete plate. The measured and predicted impact sound pressure levels are displayed in Tab.1.

Tab.1. predicted and measured impact sound pressure level

\begin{tabular}{|l|l|l|l|l|l|l|}
\hline Frequency(Hz) & 125 & 250 & 500 & 1000 & 2000 & 4000 \\
\hline Prediction without interlayer (dB) & 73.2 & 77.4 & 74.1 & 66.9 & 53.2 & 43.6 \\
\hline Measurement without interlayer (dB) & 71.8 & 76.1 & 75.7 & 68.5 & 52.4 & 41.6 \\
\hline Prediction with interlayer (dB) & 59.8 & 58.1 & 49 & 43.2 & 40.6 & 38.6 \\
\hline Measurement with interlayer (dB) & 58.3 & 56.4 & 50.3 & 45.2 & 41.5 & 40.2 \\
\hline
\end{tabular}

By comparison between predicted and measured SPL, There exist a good agreement between prediction and measurement. As the resilient interlay applied in the floating floor, it also reveals that there is an obvious reduction of impact sound pressure level in Tab. 1.

\section{Conclusions}

Sound insulation effect of resilient interlayer of a floating floor have been implemented, it is found that floating floor is an effective structure to isolate impact sound, and the resilient interlayer play a significant role in the sound transmission through the stud path, which is a dominant path in a floating floor structure. A impact sound transmission modeling approach that improves sound insulation predictions for a floating floor in the form of the option of the interlayer. The sound transmission loss through the stud path decreases with the elastic stiffness of a interlayer. Thus, the sound bridge effect is abated due to the insulation of the interlayer. As to the thickness of a interlayer, there exist different behavior at different frequency range, the thickness almost has no 
effect on sound transmission loss below the transition frequency; whereas, the sound transmission loss increases slightly with the thickness above the transition frequency. With respect to the density of a interlayer, the sound transmission loss decrease with the density of interlayer. It shows that low-density interlayer might be beneficial to sound insulation of a floating floor structure.

The comparisons between predicted and measured sound pressure level in the rooms below the floating floors with or without resilient interlayer are carried out. Without resilient interlayer, the stud path develops the dominant sound transmission path in a floating floor results from the sound bridge effect; On the contrary, the cavity path replaces the stud path as the dominant path. So, the resilient interlayer has an obvious effect to eliminate the sound bridge of a stud and ameliorate sound insulation of a floating floor. The results also present a reasonable agreement between measured and predicted value. Therefore, the technique of SEA can be employed to evaluates the impact sound transmission across a floating floor.

\section{Acknowledgement}

This research was supported by the National Natural Science Foundation of China (Project No. 51568003), the Natural Science Foundation of Guangxi Province, China(Project Nos. 2014GXNSFAA118017 and 2015GXNSFAA139254), the Systematic Project of Guangxi Key Laboratory of Disaster Prevention and Structural Safety (Project No. 2013ZDX09), and Project of Guangxi Natural Science Foundation (Project No. 2014GXNSFAA118017).

\section{References}

[1]Yanhua Liu, Qin Cao, Xiaojin Gao, The urban sound environmental quality condition and analysis of China[J], China National Environmental Monitoring Centre, 2005, 21(3): 71-72.

[2] Dean Xie, Yanxun Chen. Investigation report of Chongqing residential noise, sound insulation [J]. Journal of Acoustics.1966, 3: 170-171.

[3] Robert Craik J. M., Alexander G. Osipov. Structural isolation of walls using elastic interlayers [J]. Applied Acoustics. 1995, 46(3): 233-249.

[4] A. Schiavi, A. Pavoni Belli, M. Corallo, F. Russo. Acoustical performance characterization of resilient materials used under floating floors in dwellings [J]. Acta Acustica united with Acustica, 2007, 93(3):477-485.

[5] Tongjun Cho. Experimental and numerical analysis of floating floor resonance and its effect on impact sound transmission [J]. Journal of Sound and Vibration. 2013, 332(25): 6552-6561.

[6] K. Kim, G. Jeong, K. Yang, J. Sohn, Correlation between dynamic stiffness of resilient materials and heavyweight impact sound reduction level [J]. Building and Environment, 2009, 44 (8):1589-1600.

[7] Hua Tan. A study on the improvement of wood floor sound insulation performance of concrete floor impact sound [J]. Building Science. 2007. 9(9): 66-69.

[8] R. J. Craik. Statistical energy analysis of sound transmission through buildings [M]. UK: Gower Press, 1996.

[9] K. Gosele. Prediction of the sound transmission loss of double partitions (without structure-borne connections). Acustica 1980; 45:218-27 (in German).

[10] M. A. Stewart. Sound transmission through a chipboard floating floor supported on a concrete slab [D]. Ph.D. thesis, Heriot-Watt University, Edinburgh, 1996. 